\title{
Emotional blunting with antidepressant treatments: \\ a survey among depressed patients
}

\author{
G. M.Goodwin, ${ }^{\text {a J. Price, }}{ }^{\text {a }}$ C. De Bodinat, ${ }^{\mathrm{b}}$ J. Laredo ${ }^{\mathrm{b}}$
}

\begin{abstract}
a. University of Oxford and Oxford Health NHS Trust, Department of Psychiatry, The Warneford Hospital, Oxford, UK

b. Institut de Recherches Internationales Servier (IRIS), Suresnes, France
\end{abstract}

Corresponding author: Prof Guy Goodwin, University Department of Psychiatry and Oxford Health NHS Trust, The Warneford Hospital, Oxford, OX3 7JX, UK e-mail: guy.goodwin@psych.ox.ac.uk

Tel: +44 (0)1865 226451; Fax: +44 (0)1865 204198

Target journal: J Affect Disord

Manuscript statistics: 3403 words, 2 figures, 2 tables

\section{Disclosures of interest}

Prof Goodwin is a NIHR Senior Investigator, holds a grant from Wellcome Trust, holds shares in P1vital and has served as consultant, advisor or CME speaker for Angelini, Compass pathways, MSD, Lundbeck (/Otsuka or /Takeda), Medscape, P1Vital, Pfizer, Servier and Shire. (last 3 years). The views expressed are those of the author(s) and not necessarily those of the NHS, the NIHR or the Department of Health. 


\section{Abstract (374 words)}

Background Emotional blunting is regularly reported in depressed patients on antidepressant treatment but its actual frequency is poorly understood. We have previously used qualitative methods to develop an appropriate scale, the Oxford Questionnaire on the Emotional Side-Effects of Antidepressants (OQESA).

Methods, Results Six hundred and sixty nine depressed patients on treatment and 150 recovered (formerly depressed) controls (aged $\geq 18$ years) participated in this internet-based survey. The rate of emotional blunting in treated depressed patients was $46 \%$, slightly more frequent in men than women (52\% versus $44 \%$ ) and in those with higher Hospital Anxiety and Depression (HAD) scale scores. There was no difference according to antidepressant agent, though it appeared less frequent with bupropion. Depressed patients with emotional blunting had much higher total blunting scores on OQESA than controls $(42.83 \pm 14.73$ versus $25.73 \pm 15.00, p<0.0001)$ and there was a correlation between total blunting score and HAD-Depression score $(r=0.521)$. Thus, those with HAD-D score $>7(n=170)$ had a higher total questionnaire score, 49.23 \pm 12.03 , than those with HAD-D score $\leq 7$ ( $n=140), 35.07 \pm 13.98$, and the difference between the two groups was highly significant. However, patients with HAD-D score $\leq 7(n=140)$ had a higher total score $(35.07 \pm 13.98)$ than the recovered controls ( $n=150)(25.73 \pm 15.00)$, and the difference between the two groups was significant.

Among the patients with emotional blunting, $37 \%$ had a negative perception of their condition and $38 \%$ positive Men reported a more negative perception than women $(p=0.008)$, and patients with a negative perception were more likely to have higher HAD scores. Higher levels of emotional blunting are associated with a more negative perception of it by the patient $(r=-0.423)$.

Limitations include self-evaluation and the modest size of the sample for detection of differences between antidepressants.

Conclusions Emotional blunting is reported by nearly half of depressed patients on antidepressants. It appears to be common to all monoaminergic antidepressants. The OQESA scores are highly correlated with HAD depression score; emotional blunting cannot be described simply as a side-effect of antidepressant, but also as a 
symptom of depression. A higher degree of emotional blunting is associated with a poorer quality of remission. The OQESA scale allows the detection of this phenomenon.

Keywords: antidepressant; depression; emotional blunting; internet survey;

Oxford Questionnaire on the Emotional Side-Effects of Antidepressants, OQESA. 


\section{Introduction}

The antidepressant efficacy of selective serotonin reuptake inhibitors (SSRIs) in the management of major depression is well established. Among the positive impacts of treatment, patients generally report that they have less emotional pain with SSRIs than they had during their depressive episode. However, many treated patients also report that they suffer from a restriction in the range of emotions that they associate with normal living, such as the ability to cry or to feel enjoyment. This wholly subjective phenomenon associated with antidepressant treatment has been variously described as emotional blunting, emotional indifference, a diminution of emotional responsiveness or sensitivity, or a sense of numbing of emotion (Opbroek et al., 2002; Price et al., 2009; Sansone and Sansone, 2010).

There are currently no large-scale epidemiological studies on emotional blunting, though it has been suggested that most practising physicians may have encountered the phenomenon (Sansone and Sansone, 2010). There are a few data suggesting that emotional blunting may be quite prevalent. The results of a survey of 161 patients who had received SSRIs for depression reported that about $20 \%$ had an "inability to cry" and 46\% had a "narrowed range of affect" (Bolling and Kohlenberg, 2004). Similarly, a cross-sectional study in 117 patients with major depressive disorder found that about $30 \%$ of patients on SSRI had some form of apathy (Fava et al., 2006).

This article describes an internet survey of patients receiving various classes of antidepressants, including SSRIs and serotonin and norepinephrine reuptake inhibitors (SNRIs), in order to estimate the prevalence of emotional blunting. The survey also examined a questionnaire designed to detect emotional blunting associated with antidepressant treatments (provisionally described as the Oxford Questionnaire on the Emotional Side-effects of antidepressants) (Price et al., 2012). Finally, we examined the impact of the level of depressive symptoms on emotional blunting. 


\section{Methods}

\subsection{Study design}

This internet-based survey was conducted via three national panels of Englishspeaking individuals aged $\geq 18$ years in Canada (Angus Reid Forum, $n=98000$ ), the USA (Springboard America, $n=66000$ ), and the UK (Springboard UK, $n=40000$ ) who had agreed to participate in consumer and medical surveys.

The questionnaire had two parts: the first to screen the targets (depressed patients with emotional blunting and recovered non treated controls) and the second (main questionnaire) to explore emotional blunting in the two panels. All together, the time needed to complete the survey was around 20 minutes. Invitations to participate were sent to 7966 (4194 in Canada, 2255 in the US and 1517 in the UK) individuals identified as having depression in an initial screening (March 2010). The survey was performed by Vision Critical (www.visioncritical.com, Paris, France) between 10 September and 8 October 2010 using the secure Sparq platform.

\subsection{Panels}

Subjects who had had a probable lifetime diagnosis of depression were identified by a single question: whether a medical professional had given them a diagnosis of depression. Among this population, a group of depressed patients was defined who had been receiving an antidepressant treatment for at least 2 months (and who were still on treatment during the survey) and were either in remission or mildly depressed as assessed by a HAD depression sub-score $\leq 12$ (Hospital Anxiety and Depression scale, auto-evaluation).

The controls, like the depressed patients, had a lifetime diagnosis of depression for which they had received antidepressant treatment, but they had stopped taking antidepressants at least 2 months previously and were in remission (HAD-D $\leq 7$ ). Patients or controls receiving additional psychotropic medications (antipsychotics, mood stabilizers or antiepileptics) were excluded from the survey. 


\subsection{Evaluations}

The treated participants with depression were asked a single standardized screening question: "To what extent have you been experiencing emotional effects of your antidepressant?". The question was qualified by the explanation: "emotional effects vary, but may include, for example, feeling emotionally "numbed" or "blunted" in some way; lacking positive emotions or negative emotions; feeling detached from the world around you; or "just not caring" about things that you used to care about)." Patients who replied "mildly," "moderately," or "severely" were asked to complete the full questionnaire (Price et al., 2012). Patients who replied "not at all" or "insignificantly" to the single standardized question were not asked to complete the full questionnaire.

The full questionnaire comprises three sections for a total of 26 items: section 1 (12 items) explores the current experience of emotional blunting; section 2 ( 8 items) relates the current experience of emotional blunting with the patient's recollection of their normal emotional state prior to their depression; and section 3 (6 items) assesses the patient's perception of a link between the antidepressant treatment and the experience of emotional blunting, and whether this has affected compliance with treatment or induced plans to discontinue. Each item is rated on a 5-point scale ranging from disagree to agree. The depressed patients completed all three sections. They were also asked to rate the impact of emotional blunting in their daily life, which was measured on a graduated scale (VAS) ranging from very negative (0) to very positive (10).

The recovered controls completed the first two sections (20 items), but not the last section, which relates to current antidepressant treatment (Price et al., 2012).

\subsection{Statistical methods}

Patients with two or more antidepressant treatments were excluded from the analyses. Descriptive statistics using mean $\pm S D$ as well as median were provided for quantitative variables, and numbers and percentages of participants per class for qualitative variables. Differences between groups on quantitative variables tested were based on a Student's t-test for independent samples or on a single one-way analysis of variance, according to the number of groups, with a type I error $\alpha$ at 5\% 
(bilateral situation). Corresponding estimate and standard error [E (SE)] of the difference between groups, as well as 95\% two-sided confidence intervals ( $\mathrm{Cl}$ ) were also provided. Correlations were based on Pearson correlation coefficient ( $r$ ). All analyses were performed using SAS 9.1 software.

\section{Results}

The distribution of participants in the survey is shown in Figure 1. A total of 7966 individuals with depression were invited to participate. Within this sample, 854 patients on antidepressants and 150 recovered controls were recruited as agreed in the contract with the survey company. In the sample on antidepressants, 401 did report significant emotional blunting, of whom 91 were receiving more than one antidepressant. The latter were excluded to provide a more homogeneous population of depressed patients on monotherapy, for whom the rate of emotional blunting was 46\% (310/669); 53\% (453/854) reported no emotional blunting on the screening question.

The populations with and without emotional blunting and on a single antidepressant agent $(n=669)$ and the recovered controls $(n=150)$ are presented in Table 1 . The phenomenon was slightly more frequent in men than in women, with $52 \%(98 / 187)$ of the men reporting emotional blunting versus $44 \%(212 / 482)$ of the women. There were no differences in age or other demographic variables for patients with or without blunting. The patients with blunting had been treated for a mean of 106.9 \pm 93.6 months (median, 84 months, range 2 to 600 months) versus $103.7 \pm 84.0$ months (median, 87 months, range 2 to 600 months) for patients without emotional blunting. The demographics of the group of recovered controls were similar to those of the depressed patients. In general, patients with emotional blunting had higher HAD scores for depression (HAD-D) and anxiety (HAD-A) than those without.

There was no difference in the rate of emotional blunting by antidepressant agent (Table 2), which was generally between $43 \%$ and $56 \%$. There were two outliers: emotional blunting appeared to be less frequent with bupropion (33\% of 40 patients 
taking bupropion reported emotional blunting) and more frequent with duloxetine (75\% of 36 patients taking duloxetine).

Scores on the blunting questionnaire

The monotherapy patients with emotional blunting had a mean total score of $42.83 \pm 14.73$, while the recovered controls had a mean of $25.73 \pm 15.00$ (median, 44.0 and 25.0, respectively), with a significant difference between the two groups [E (SE), 17.11 (1.47), 95\% Cl, 14.21 to $20.00, p<0.0001$ ] (Fig. 2). Among the monotherapy patients with emotional blunting, those with HAD-D score $>7(n=170)$ had a higher total questionnaire score, $49.23 \pm 12.03$, than those with HAD-D score $\leq 7$ ( $n=140)$, $35.07 \pm 13.98$, and the difference between the two groups was highly significant $[E$ (SE), 14.16 (1.48), 95\% Cl, 11.25 to 17.07, p<0.0001]. However, patients with HAD-D score $\leq 7(n=140)$ had a higher total score $(35.07 \pm 13.98)$ than the recovered controls ( $n=150)(25.73 \pm 15.00)$, and the difference between the two groups was significant [E (SE), 9.34 (1.71), $95 \% \mathrm{Cl}, 5.99$ to $12.70, \mathrm{p}<0.0001$.

Table 2 shows the blunting scores in different domains identified previously in the OQESA. The 'General reduction' or GR is most clearly associated with current antidepressant monotherapy. Emotional detachment is largely accounted for by differences in HAD-D depression scores. Not Caring and Positive reductions appear related to both HAD-D scores and current depression.

There was a correlation between the total score and the HAD-D score (Pearson correlation coefficient, $r=0.521)$, but not the HAD-A score $(r=0.139)$. No correlation was found between the total score and age $(r=-0.041)$ or duration of antidepressant treatment $(r=-0.148)$ and the total score was not different between three educational levels. Among the 310 patients with emotional blunting, 114 (37\%) had a negative perception of their condition (less than 5 on the VAS), $118(38 \%)$ had a positive perception (more than 5 on the VAS), and $78(25 \%)$ neutral ( 5 on the VAS)

(Figure 3). There was a significant difference between perception of emotional blunting in men and women, with men reporting a more negative perception than women [mean VAS, $4.7 \pm 2.0$ men versus $5.3 \pm 1.9$ for women, $E(S E), 0.64(0.24), 95 \%$ $\mathrm{Cl}, 0.17$ to $1.11, \mathrm{p}=0.008]$. The patients with a very negative perception were more 
likely to have higher HAD-D and HAD-A scores (9.5 \pm 2.3 and $11.7 \pm 4.0$, respectively, for patients with less than 4 on the VAS, $n=65$ ) than the patients with a very positive perception of their emotional blunting $(6.4 \pm 2.8$ and $8.9 \pm 3.8$ for patients with more than 6 on the VAS, $n=83$ ). There was a slight correlation between HAD-D score and perception of emotional blunting (Pearson correlation coefficient, $r=-0.389$ ), but not between HAD-A and perception of emotional blunting ( $r=-0.239)$. A higher blunting score was correlated with a more negative perception of emotional blunting on the VAS (Pearson correlation coefficient, $r=-0.423$ ). There was no correlation between perception of emotional blunting and age $(r=0.06)$ or length of treatment with antidepressants $(r=0.139)$.

\section{Discussion}

The results of this internet survey suggest that emotional blunting may be experienced by nearly half of patients (46\%) on SSRIs, SNRIs, or tricyclic antidepressants. Our results provide the first quantitative description of this condition in a community sample, and are in accord with rates suggested in previous studies (Opbroek et al., 2002; Bolling and Kohlenberg, 2004; Fava et al., 2006). We found it to be slightly more frequent in male patients, and was correlated with higher HAD scores, i.e. the presence of more symptoms of depression. Of those reporting emotional blunting, $37 \%$ had a negative perception of the condition and $38 \%$ a positive perception.

The questionnaire was systematically constructed on the basis of qualitative assessments of emotional blunting in patients on SSRIs using data collected in individual interviews and from patient websites (Price et al., 2009). The implicit attribution of the experiences to current medication was reflected in its original name: The Oxford Questionnaire on the Emotional Side-Effects of Antidepressants (OQESA). It has been tested on a previous sample of depressed patients, and appears to be acceptable to patients, and have high construct validity and reliability (Price et al., 2012). There was a highly significant difference in scale score for patients identified with emotional blunting and the recovered control group, not taking antidepressants, in the present study $(p<0.0001)$. Moreover, this difference was also 
present when treated patients and controls were matched for current depressive symptoms. Blunting is, therefore, associated with antidepressant treatment as expected.

However, there is also an association between current depressive symptoms and emotional blunting. We found correlations between the severity of the depressive symptoms on the HAD-D scale and the blunting scale score $(r=-0.521)$. The strong association with depression means we believe the description of the scale should be neutral in relation to the causes of emotional blunting which may be multiple. Indeed, the scale remains a work in progress requiring more data collected under double blind conditions before we can be confident of the causes of sub-domains shown in Table 2. The obvious prevailing hypothesis, that it is a side-effect of antidepressants would predict differences between antidepressants. This was partly confirmed here by the low rates of blunting in bupropion-treated patients. However, if emotional blunting is also a component of depressed mood, its association with particular antidepressants, in particular the SSRIs could reflect their failure to treat this kind of symptom as much as a side effect of the drug per se.

Higher levels of emotional blunting are associated with a more negative perception of it by the patient ( $r=-0.423)$. Thus, our results suggest that a high degree of emotional blunting is associated with a poorer quality of remission in depressive symptoms and a more negative perception of the condition, and may be a reason for treatment cessation. By contrast, the presence of a low degree of emotional blunting appears to be acceptable (or even preferable) to depressed patients. This is in line with qualitative reports of the condition (Price et al., 2009), in which some patients described their emotional blunting as "unhelpful" and some "helpful".

Loss of interest and pleasure is known to be part of the endophenotype of depression (McCabe et al., 2009), and studies in healthy volunteers have demonstrated that treatment with SSRIs is associated with diminished neural responses to reward and aversive stimuli. There is evidence that decreased positive affect is linked to a dysregulation of dopaminergic and noradrenergic 
neurotransmission, but not the serotonergic pathways. Treatment with SSRIs enhances serotonergic neurotransmission, and may blunt dopaminergic and noradrenergic activity, and it has been suggested that this could explain the symptoms such as anhedonia and fatigue often seen after depressed patients present an overall positive response to a SSRI (Blier and Briley, 2011). In line with this, the agent associated with the lowest rate of emotional blunting, bupropion (33\%), is a dopamine and norepinephrine reuptake inhibitor and releaser and does not affect the serotonergic system. It could be concluded therefore, that the phenomenon of emotional blunting is not restricted to SSRIs, but is to be common to all monoaminergic antidepressants.

In this context, we previously suggested whether emotional blunting is a residual symptom could be tested by evaluating healthy volunteers taking antidepressants (Price et al., 2012). The absence of an effect would confirm blunting as a residual symptom of the depressed state that is either not addressed by antidepressant treatment or is the consequence of an interaction between SSRI action and the mildly depressed state. It is unlikely to be a simple side effect of treatment per se.

Our study has a number of limitations. First, the diagnosis may not be as reliable as in clinical studies when the patients are interviewed by a psychiatrist and the presence of comorbidities is difficult to exclude. However, the relatively large sample size helps generalizability and exclusion of patients receiving antipsychotics or mood stabilizers will have helped to exclude major comorbidities. Second, the self-evaluation of depression on the internet is becoming established, but is subject to reservations in regard to the potential for spurious data entry and the use of scales developed for pencil and paper. The present sample is taken from a registered cohort which helps to ensure entry of valid data. The HAD scale has important advantages as it is simple to use and has screening properties similar to other more comprehensive instruments (Bjelland et al., 2002). Third, the absence of validated versions of the blunting scale in other languages restricted the survey to Englishspeaking countries. Fourth, a much larger sample would be required to detect differences between the many antidepressants in current use and even differences 
between various classes, for example, SSRI and SNRIs. Finally, internet surveys are likely to be biased towards younger populations with poor representation of the elderly.

\section{Conclusion}

Emotional blunting is reported by nearly half of patients on SSRI, SNRI, or tricyclic antidepressants. The phenomenon of emotional blunting is not restricted to SSRIs, but may require a serotonergic effect. The presence of emotional blunting correlates with a higher HAD-D score, and may be a residual symptom of depression rather than simply a side effect of treatment. The blunting scale we have previously described as the OQESA is a valid scale for measuring this phenomenon. 
Bjelland I, Dahl AA, Haug TT, Neckelmann D (2002) The validity of the Hospital Anxiety and Depression Scale. An updated literature review. J Psychosom Res 52: 69-77.

Blier P, Briley M (2011) The noradrenergic symptom cluster: clinical expression and neuropharmacology. Neuropsychiatr Dis Treat 7: 15-20.

Bolling MY, Kohlenberg RJ (2004) Reasons for quitting serotonin reuptake inhibitor therapy: paradoxical psychological side effects and patient satisfaction. Psychother Psychosom 73: 380-385.

Fava M, Graves LM, Benazzi F, Scalia MJ et al. (2006) A cross-sectional study of the prevalence of cognitive and physical symptoms during long-term antidepressant treatment. J Clin Psychiatry 67: 1754-1759.

McCabe C, Cowen PJ, Harmer CJ (2009) Neural representation of reward in recovered depressed patients. Psychopharmacology (Berl) 205: 667-677.

McCabe C, Mishor Z, Cowen PJ, Harmer CJ (2010) Diminished neural processing of aversive and rewarding stimuli during selective serotonin reuptake inhibitor treatment. Biol Psychiatry 67: 439445.

Nutt D, Demyttenaere K, Janka Z, Aarre T et al. (2007) The other face of depression, reduced positive affect: the role of catecholamines in causation and cure. J Psychopharmacol 21: 461-471.

Opbroek A, Delgado PL, Laukes C, McGahuey C et al. (2002) Emotional blunting associated with SSRIinduced sexual dysfunction. Do SSRIs inhibit emotional responses? Int J Neuropsychopharmacol 5: 147-151.

Price J, Cole V, Doll H, Goodwin GM (2012) The Oxford Questionnaire on the Emotional Side-Effects of Antidepressants: Development validity, reliability and sensitivity to change. J Affect Disord 140, 6674 .

Price J, Cole V, Goodwin GM (2009) Emotional side-effects of selective serotonin reuptake inhibitors: qualitative study. Br J Psychiatry 195: 211-217.

Sansone RA, Sansone LA (2010) SSRI-Induced Indifference. Psychiatry (Edgmont) 7: 14-18.

Schmidt K, Nolte-Zenker B, Patzer J, Bauer M et al. (2001) Psychopathological correlates of reduced dopamine receptor sensitivity in depression, schizophrenia, and opiate and alcohol dependence. Pharmacopsychiatry 34: 66-72. 
Figure 1. Distribution of patients in the survey.

HAD-D, Hospital Anxiety and Depression scale (depression sub-score).

Figure 2. OQESA scores in depressed patients and recovered controls.

Figure 3. Patients' perception of emotional blunting on a visual analogue scale (VAS) in depressed patients with one antidepressant $(n=310)$. 
Table 1. Characteristics of participants in the survey: depressed patients with monotherapy and recovered controls.

HAD-D, Hospital Anxiety and Depression scale (depression subscore).

HAD-A, Hospital Anxiety and Depression scale (anxiety subscore).

\begin{tabular}{lccc}
\hline & \multicolumn{2}{c}{$\begin{array}{c}\text { Depressed patients on one antidepressant } \\
(\mathbf{n = 6 6 9 )}\end{array}$} & $\begin{array}{c}\text { Recovered controls } \\
(\mathbf{n = 1 5 0 ) *}\end{array}$ \\
\cline { 2 - 3 } & $\begin{array}{c}\text { Emotional blunting } \\
(\mathbf{n = 3 1 0 )}\end{array}$ & $\begin{array}{c}\text { No emotional } \\
\text { blunting }(\mathbf{n = 3 5 9 )}\end{array}$ & \\
\hline Male (\%) & $98(32 \%)$ & $89(25 \%)$ & $46(31 \%)$ \\
Female (\%) & $212(68 \%)$ & $270(75 \%)$ & $104(69 \%)$ \\
Age (mean \pm SD) & $49.5 \pm 12.3$ & $51.7 \pm 12.4$ & $44.4 \pm 14.2$ \\
Working (\%) & $147(47 \%)$ & $182(51 \%)$ & $87(58 \%)$ \\
HAD-D score & $7.9 \pm 2.8$ & $4.8 \pm 3.3$ & $3.9 \pm 2.2$ \\
HAD-A score & $10.1 \pm 3.9$ & $7.4 \pm 3.8$ & $7.8 \pm 3.9$ \\
\hline
\end{tabular}

*Mean time since cessation of antidepressant treatment, $27.7 \pm 20.7$ months (range, 3 to 72 months). 
Table 3. Scores for different domains of emotional blunting in monotherapy patients with depressive symptoms or in remission (HAD-D $<7)$ and recovered controls

\begin{tabular}{|c|c|c|c|}
\hline \multirow[b]{2}{*}{$\begin{array}{l}\text { Mean } \pm \text { SD } \\
\text { Median }\end{array}$} & \multicolumn{2}{|c|}{ Emotionaly blunted patients } & \multirow{2}{*}{$\begin{array}{c}\text { Recovered controls } \\
\text { HAD-D } \leq 7 \\
(\mathrm{n}=150)\end{array}$} \\
\hline & $\begin{array}{c}7<\text { HAD-D } \leq 12 \\
(\mathrm{n}=170)\end{array}$ & $\begin{array}{c}\text { HAD-D } \leq 7 \\
(\mathrm{n}=140)\end{array}$ & \\
\hline OQESA Total Score & $\begin{array}{c}49.2 \pm 12.0 \\
50\end{array}$ & $\begin{array}{c}35.1 \pm 14.0 \\
34\end{array}$ & $\begin{array}{c}25.7 \pm 15.0 \\
25\end{array}$ \\
\hline General Reduction (GR) & $\begin{array}{c}13.2 \pm 3.9 \\
13\end{array}$ & $\begin{array}{c}11.2 \pm 4.2 \\
11\end{array}$ & $\begin{array}{c}6.8 \pm 4.6 \\
7\end{array}$ \\
\hline Emotional detachment (ED) & $\begin{array}{c}8.5 \pm 5.7 \\
8\end{array}$ & $\begin{array}{c}5.6 \pm 5.1 \\
4\end{array}$ & $\begin{array}{c}5.0 \pm 4.8 \\
4\end{array}$ \\
\hline Not Caring (NC) & $\begin{array}{c}12 \pm 3.5 \\
12\end{array}$ & $\begin{array}{c}7.7 \pm 4.3 \\
8\end{array}$ & $\begin{array}{c}6.2 \pm 4.0 \\
6\end{array}$ \\
\hline Positive Reduction (PR) & $\begin{array}{c}15.6 \pm 3.4 \\
16\end{array}$ & $\begin{array}{c}10.6 \pm 4.9 \\
11\end{array}$ & $\begin{array}{c}7.8 \pm 5.4 \\
8\end{array}$ \\
\hline
\end{tabular}


Table 2. Presence of emotional blunting according to antidepressant agent, evaluated in depressed patients with monotherapy using a single standardized question (see Methods).

\begin{tabular}{lcc}
\hline Antidepressant & $\begin{array}{c}\text { Patients receiving } \\
\text { antidepressant, } \mathbf{n}\end{array}$ & $\begin{array}{c}\text { Patients with emotional } \\
\text { blunting, } \mathbf{n}(\%)\end{array}$ \\
\hline Citalopram & 127 & $58(46 \%)$ \\
Venlafaxine & 105 & $48(46 \%)$ \\
Fluoxetine & 98 & $46(47 \%)$ \\
Sertraline & 80 & $36(45 \%)$ \\
Paroxetine & 58 & $25(43 \%)$ \\
Escitalopram & 53 & $23(43 \%)$ \\
Bupropion & 40 & $13(33 \%)$ \\
Duloxetine & 36 & $27(75 \%)$ \\
Amitriptyline & 17 & $8(47 \%)$ \\
Mirtazapine & 17 & $7(42 \%)$ \\
Desvenlafaxine & 9 & $5(56 \%)$ \\
Others & 29 & $14(48 \%)$ \\
Total & 669 & $310(46 \%)$ \\
\hline
\end{tabular}

